\title{
Transitions Between Bursting Modes in the Integrated Oscillator Model for Pancreatic $\beta$-cells
}

\author{
Isabella Marinelli \\ Basque Center for Applied Mathematics \\ Bilbao Basque Country \\ Spain \\ Theodore Vo \\ Florida State University \\ Department of Mathematics \\ Tallahassee, FL \\ Luca Gerardo-Giorda \\ Basque Center for Applied Mathematics \\ Bilbao Basque Country \\ Spain \\ Richard Bertram \\ Florida State University \\ Department of Mathematics and \\ Programs in Neuroscience and Molecular Biophysics \\ Tallahassee, FL \\ July 17,2018
}




\begin{abstract}
Insulin-secreting $\beta$-cells of pancreatic islets of Langerhans produce bursts of electrical impulses, resulting in intracellular $\mathrm{Ca}^{2+}$ oscillations and pulsatile insulin secretion. The mechanism for this bursting activity has been the focus of mathematical modeling for more than three decades, and as new data are acquired old models are modified and new models are developed. Comprehensive models must now account for the various modes of bursting observed in islet $\beta$-cells, which include fast bursting, slow bursting, and compound bursting. One such model is the Integrated Oscillator Model (IOM), in which $\beta$-cell electrical activity, intracellular $\mathrm{Ca}^{2+}$, and glucose metabolism interact via numerous feedforward and feedback pathways. These interactions can produce metabolic oscillations with a sawtooth time course or a pulsatile time course, reflecting very different oscillation mechanisms. In this report, we determine conditions favorable to one form of oscillations or the other, and examine the transitions between modes of bursting and the relationship of the transitions to the patterns of metabolic oscillations. Importantly, this work clarifies what can be expected in experimental measurements of $\beta$-cell oscillatory activity, and suggests pathways through which oscillations of one type can be converted to oscillations of another type.
\end{abstract}




\section{Introduction}

Insulin is secreted by $\beta$-cells that, in mammals, make up the majority of the cells in pancreatic micro-organs called islets of Langerhans [19]. This hormone is essential for glucose uptake by muscle and fat cells, and dysfunction or loss of $\beta$-cell mass is responsible for abnormal blood glucose homeostasis and diabetes (see [35] for review). As with the secretion of many other hormones, insulin secretion is pulsatile [32]. Early reports of peripheral insulin measurements in fasted humans found oscillations with a period of approximately 15 min [24], but more recent reports using improved detection methods and measurements of insulin in the hepatic portal circulation from rodents [26], pigs [22], and humans [39] show oscillations with periods of 4-6 min. In addition to these oscillations, slower ultradian oscillations with periods of 1 to 2 hours have been observed in humans [40]. The liver plays a key role in glucose homeostasis by releasing glucose between meals and suppressing hepatic glucose production during and shortly after meals. A number of studies have demonstrated that insulin pulses, rather than constant insulin, facilitate the liver's response to the insulin $[9,25]$. The clinical importance of insulin oscillations has been established by studies showing that the regularity of insulin oscillations is disrupted in patients with type II diabetes and their near relatives [23, 33].

The mechanism for insulin oscillations has been debated for decades [35]. A central question in this debate is whether the electrical bursting and downstream insulin pulses are driven by oscillations in glucose metabolism or whether they are driven by $\mathrm{Ca}^{2+}$ feedback onto ATP production/hydrolysis or onto $\mathrm{Ca}^{2+}$-activated $\mathrm{K}^{+}$channels. Following the publication of the first mathematical model of bursting in $\beta$-cells in 1983 [11], numerous models have

been developed that reflect one of these paradigms $[3,4,6,10,13,18,21,38]$. A recent 
model, called the Integrated Oscillator Model (IOM), is a hybrid in which $\mathrm{Ca}^{2+}$ feedback onto an enzyme at the end of glycolysis can be a key factor in driving oscillations, but in which oscillations in glycolysis can also occur independently of $\mathrm{Ca}^{2+}$ feedback [27].

In a companion paper [28], we employed a fast-slow analysis of the IOM to uncover the details of the bursting mechanisms, but only touched on the question of which conditions are necessary for bursting to be driven by glycolytic oscillations or by the feedback of $\mathrm{Ca}^{2+}$ onto glycolysis. We address the question in this report. In particular, we determine values of key parameters for which intrinsic glycolytic oscillations occur, and values for which bursting oscillations are a product of $\mathrm{Ca}^{2+}$ feedback onto glycolysis. The distinct behaviors are found in overlapping regions of parameter space, and we analyze the transitions that occur between the different regions. The parameters varied in the examination are the maximal conductance of the $\mathrm{Ca}^{2+}$-activated $\mathrm{K}^{+}$current $\left(g_{\mathrm{K}(\mathrm{Ca})}\right)$ and the maximum flux rate through the pyruvate dehydrogenase reaction $\left(v_{\mathrm{PDH}}\right)$. They were chosen because of their pivotal roles in the electrical and metabolic portions of the model, respectively.

The origin of $\beta$-cell oscillations, whether due to intrinsic glycolytic oscillations or $\mathrm{Ca}^{2+}$ feedback, is important to know when interpreting the many experimental findings that have been published about $\beta$-cell oscillations. For example, numerous in vitro islet recordings of compound bursting electrical activity or compound $\mathrm{Ca}^{2+}$ oscillations have been published $[1,7,12,20,45]$. These oscillations consist of fast bursts (usually less than 30 sec in duration) clustered into episodes (duration of several minutes), with each episode separated by a long (two or three minutes) silent phase. Figure 1 depicts representative slow (A), fast (B), and compound (C) bursting rhythms. Compound oscillations are thus a composition of fast and slow oscillations. 


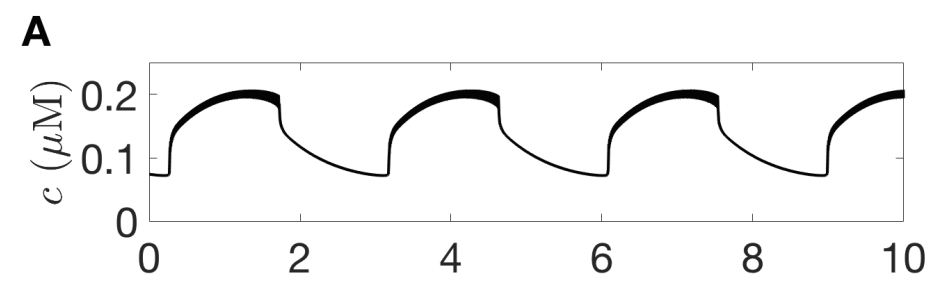

B
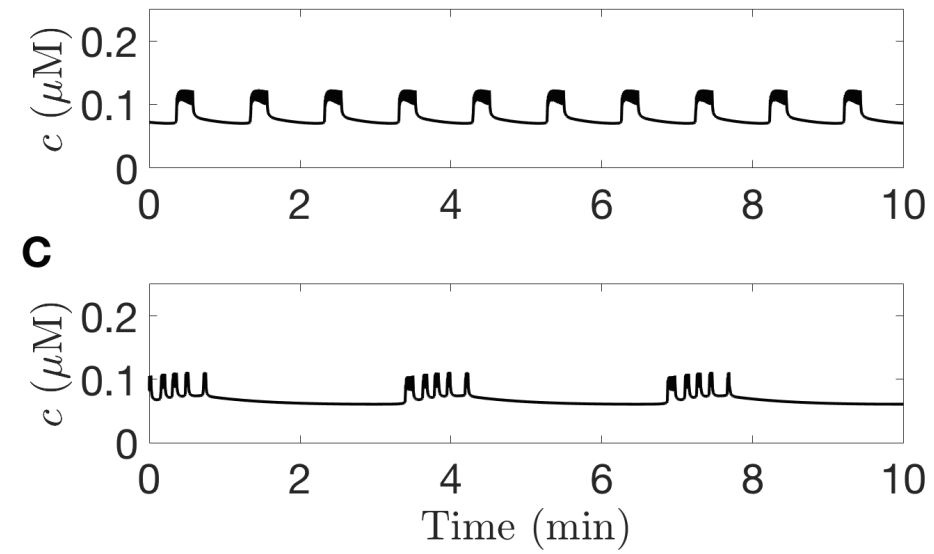

Figure 1: Representative slow (A), fast (B), and compound (C) bursting rhythms. These plots show cytosolic $\mathrm{Ca}^{2+}$ time courses obtained by implementing the IOM described in the Mathematical Model section.

Do these compound $\mathrm{Ca}^{2+}$ oscillations reflect glycolytic oscillations, or are they essential to the burst mechanism? As another example, measurements of the glycolytic metabolite fructose 1,6-bisphosphate (FBP) during slow bursting showed a sawtooth pattern, with a slow rise during the silent phase of a burst and a slow decline during the active or spiking phase [31]. Is this pattern, which reflects oscillations driven by $\mathrm{Ca}^{2+}$ feedback onto glycolysis, always expected during slow bursting oscillations? In this paper, we show that compound bursting is always driven by glycolytic oscillations, whereas slow bursting can reflect either self-sustained glycolytic oscillations or $\mathrm{Ca}^{2+}$ feedback onto glycolysis. Furthermore, we show that the FBP concentration can have either a pulsatile or sawtooth pattern during slow 
bursting, and this time course serves as a fingerprint for the oscillation mechanism.

\section{Mathematical Model}

In this article, we study a modified version of the IOM, consisting of two modules. The first module describes the $\beta$-cell electrical activity and $\mathrm{Ca}^{2+}$ dynamics. The second module describes the production and utilization of glycolysis and adenosine triphosphate (ATP). Computer codes for the model can be downloaded from www.math.fsu.edu/ bertram/software/islet.

\section{The Electrical and Calcium Module}

The $\beta$-cell electrical activity is described with a conductance-based model, where the rate of change of the cell's membrane potential $(V)$ is determined by

$$
\frac{d V}{d t}=-\frac{1}{C}\left[I_{\mathrm{Ca}}+I_{\mathrm{K}}+I_{\mathrm{K}(\mathrm{Ca})}+I_{\mathrm{K}(\mathrm{ATP})}\right],
$$

where $C$ is the membrane capacitance, $I_{\mathrm{Ca}}$ is a $\mathrm{Ca}^{2+}$ current responsible for the upstroke of action potentials, $I_{\mathrm{K}}$ is a delayed-rectifying $\mathrm{K}^{+}$current responsible for the downstroke of action potentials, $I_{\mathrm{K}(\mathrm{Ca})}$ is a $\mathrm{Ca}^{2+}$-activated $\mathrm{K}^{+}$current that plays a key role in packaging action potentials into bursts, and $I_{\mathrm{K}(\mathrm{ATP})}$ is a $\mathrm{K}^{+}$current that is inactivated by ATP and is thereby regulated by the glucose level.

The $\mathrm{Ca}^{2+}$ current is assumed to change instantaneously with changes in $V$, and is described by

$$
I_{\mathrm{Ca}}=g_{\mathrm{Ca}} m_{\infty}(V)\left(V-V_{\mathrm{Ca}}\right),
$$


where $g_{\mathrm{Ca}}$ is the maximal conductance, $V_{\mathrm{Ca}}$ is the $\mathrm{Ca}^{2+}$ Nernst potential, and $m_{\infty}$ is the activation function given by the increasing sigmoidal

$$
m_{\infty}(V)=\frac{1}{1+\exp \left[\left(\nu_{m}-V\right) / s_{m}\right]}
$$

Values for the shape parameters $\nu_{m}$ and $s_{m}$, as well as other parameters, are given in Table 1.

The activation, $n$, of the delayed rectifying $\mathrm{K}^{+}$current has the first-order kinetics

$$
\frac{d n}{d t}=\frac{n_{\infty}(V)-n}{\tau_{n}}
$$

where $n_{\infty}(V)$ is a sigmoid of the form (2), but with shape parameters $\nu_{n}$ and $s_{n}$. The delayed rectifying current is

$$
I_{\mathrm{K}}=g_{\mathrm{K}} n\left(V-V_{\mathrm{K}}\right) \text {. }
$$

Activation of the $\mathrm{K}(\mathrm{Ca})$ current is through binding of $\mathrm{Ca}^{2+}$ ions to intracellular binding sites with activation function $q_{\infty}(c)$, where $c$ is the free intracellular $\mathrm{Ca}^{2+}$ concentration. The $\mathrm{K}(\mathrm{Ca})$ current is then

$$
I_{\mathrm{K}(\mathrm{Ca})}=g_{\mathrm{K}(\mathrm{Ca})} q_{\infty}(c)\left(V-V_{\mathrm{K}}\right)
$$

where

$$
q_{\infty}(c)=\frac{c^{2}}{k_{d}^{2}+c^{2}} .
$$

The maximal conductance, $g_{\mathrm{K}(\mathrm{Ca})}$, is one of the key parameters that we vary to explore the types of electrical bursting produced by the model. 


\begin{tabular}{|l|l|l|}
\hline$C=5300 \mathrm{fF}$ & $g_{\mathrm{Ca}}=1000 \mathrm{pS}$ & $g_{\mathrm{K}}=2700 \mathrm{pS}$ \\
$g_{\mathrm{K}(\mathrm{Ca})}=$ varies & $g_{\mathrm{K}(\mathrm{ATP})}=25000 \mathrm{pS}$ & $V_{\mathrm{Ca}}=25 \mathrm{mV}$ \\
$V_{\mathrm{K}}=-75 \mathrm{mV}$ & $\nu_{m}=-20 \mathrm{mV}$ & $s_{m}=12 \mathrm{mV}$ \\
$\nu_{n}=-16 \mathrm{mV}$ & $s_{n}=5 \mathrm{mV}$ & $\tau_{n}=20 \mathrm{~ms}$ \\
$k_{d}=0.5 \mu \mathrm{M}$ & $k_{d d}=17 \mu \mathrm{M}$ & $k_{t t}=1 \mu \mathrm{M}$ \\
$k_{t d}=26 \mu \mathrm{M}$ & $f_{\mathrm{Ca}}=0.01$ & $\alpha=5.18 \times 10^{-18} \mu \mathrm{mol} \mathrm{fA}^{-1} \mathrm{~ms}^{-1}$ \\
$V_{\text {cyt }}=1.15 \times 10^{-12} 1$ & $k_{\mathrm{PMCA}}=0.2 \mathrm{~ms}^{-1}$ & $k_{\mathrm{SERCA}}=0.4 \mathrm{~ms}^{-1}$ \\
$p_{\text {leak }}=2 \times 10^{-4} \mathrm{~ms}^{-1}$ & $k_{\text {uni }}=0.4 \mathrm{~ms}^{-1}$ & $k_{\mathrm{NaCa}}=0.001 \mathrm{~ms}^{-1}$ \\
$\sigma_{\mathrm{m}}=100$ & $\sigma_{\mathrm{er}}=31$ & \\
\hline
\end{tabular}

Table 1: Parameters for the electrical/calcium module.

The final current is described by

$$
I_{\mathrm{K}(\mathrm{ATP})}=g_{\mathrm{K}(\mathrm{ATP})} o_{\infty}(\mathrm{ADP}, \mathrm{ATP})\left(V-V_{\mathrm{K}}\right)
$$

where the activation function, $o_{\infty}$, depends on the concentrations of cytosolic adenosine diphosphate (ADP) as well as ATP, and is given by

$$
o_{\infty}(\mathrm{ADP}, \mathrm{ATP})=\frac{0.08+0.89\left(\frac{\mathrm{MgADP}}{k_{d d}}\right)^{2}+0.16\left(\frac{\mathrm{MgADP}}{k_{d d}}\right)}{\left(1+\frac{\mathrm{MgADP}}{k_{d d}}\right)^{2}\left(1+\frac{\mathrm{ATP}^{4-}}{k_{t t}}+\frac{\mathrm{ADP}^{3-}}{k_{t d}}\right)}
$$

Here, $\mathrm{MgADP}=0.165 \mathrm{ADP}, \mathrm{ADP}^{3-}=0.135 \mathrm{ADP}$, and $\mathrm{ATP}^{4-}=0.05 \mathrm{ATP}$.

During action potentials $\mathrm{Ca}^{2+}$ enters the cell through $\mathrm{Ca}^{2+}$ channels and is removed from the cell through pumps in the plasma membrane. The $\mathrm{Ca}^{2+}$ also flows into and out of the mitochondria and endoplasmic reticulum (ER). The dynamics of the free cytosolic $\mathrm{Ca}^{2+}$ concentration are given by

$$
\frac{d c}{d t}=f_{\mathrm{Ca}}\left(J_{\mathrm{mem}}-J_{\mathrm{m}}-J_{\mathrm{er}}\right)
$$




\begin{tabular}{|l|l|l|l|}
\hline$J_{\mathrm{GK}}=0.001 \mu \mathrm{M} \mathrm{m}^{-1}$ & $v_{\mathrm{PFK}}=0.01 \mu \mathrm{M} \mathrm{ms}^{-1}$ & $k_{\mathrm{PFK}}=0.06$ & $K_{1}=30 \mu \mathrm{M}$ \\
$K_{2}=1 \mu \mathrm{M}$ & $K_{3}=5 \times 10^{4} \mu \mathrm{M}$ & $K_{4}=10^{3} \mu \mathrm{M}$ & $f_{13}=0.02$ \\
$f_{23}=0.2$ & $f_{41}=20$ & $f_{42}=20$ & $f_{43}=20$ \\
$v_{\mathrm{PDH}}=$ varies & $K_{\mathrm{PDH}}=200 \mu \mathrm{M}$ & $\tau_{a}=300000 \mathrm{~ms}$ & $A_{\mathrm{tot}}=3000 \mu \mathrm{M}$ \\
\hline
\end{tabular}

Table 2: Parameters for the metabolic module.

where $f_{\mathrm{Ca}}$ is the fraction of $\mathrm{Ca}^{2+}$ ions not bound to buffers, and $J_{\mathrm{mem}}, J_{\mathrm{m}}$, and $J_{\text {er }}$ represent the $\mathrm{Ca}^{2+}$ flux densities across the plasma membrane, into the mitochondria, and into the ER, respectively. They are described by

$$
\begin{aligned}
J_{\mathrm{mem}} & =-\left[\frac{\alpha}{V_{\mathrm{cyt}}} I_{\mathrm{Ca}}+k_{\mathrm{PMCA}} c\right], \\
J_{\mathrm{er}} & =k_{\mathrm{SERCA}} c-p_{\text {leak }}\left(c_{\mathrm{er}}-c\right), \\
J_{\mathrm{m}} & =k_{\mathrm{uni}} c-k_{\mathrm{NaCa}}\left(c_{\mathrm{m}}-c\right) .
\end{aligned}
$$

The parameter $V_{\text {cyt }}$ is the volume of the cytosolic compartment and $\alpha$ converts current to ion flux. The $k$ parameters reflect the strength of $\mathrm{Ca}^{2+}$ pumps or $\mathrm{Na}^{+}-\mathrm{Ca}^{2+}$ exchanger, while $p_{\text {leak }}$ reflects the leak of $\mathrm{Ca}^{2+}$ across the ER membrane.

The free $\mathrm{Ca}^{2+}$ concentration in the mitochondria and ER are described by

$$
\begin{aligned}
\frac{d c_{\mathrm{m}}}{d t} & =f_{\mathrm{Ca}} \sigma_{\mathrm{m}} J_{\mathrm{m}}, \\
\frac{d c_{\mathrm{er}}}{d t} & =f_{\mathrm{Ca}} \sigma_{\mathrm{er}} J_{\mathrm{er}},
\end{aligned}
$$

where the $\sigma$ parameters are ratios of cytosolic volume to ER or mitochondria volumes. 


\section{The Metabolic Module}

For the metabolic module, we use a simplified model that focuses on the allosteric enzyme phosphofructokinase (PFK), which is the basis for oscillations in glycolysis [37]. Parameter values for the model are given in Table 2. The cytosolic concentrations of the enzyme's substrate fructose 6-phosphate (F6P) and product fructose 1,6-bisphosphate (FBP) are described by

$$
\begin{aligned}
& \frac{d \mathrm{~F} 6 \mathrm{P}}{d t}=0.3\left(J_{\mathrm{GK}}-J_{\mathrm{PFK}}\right), \\
& \frac{d \mathrm{FBP}}{d t}=J_{\mathrm{PFK}}-\frac{1}{2} J_{\mathrm{PDH}},
\end{aligned}
$$

where the parameter $J_{\mathrm{GK}}$ is the glucose-dependent glucokinase reaction rate, $J_{\mathrm{PFK}}$ is the PFK reaction rate, and $J_{\mathrm{PDH}}$ is the pyruvate dehydrogenase $(\mathrm{PDH})$ reaction rate. The complicated PFK rate depends on its substrate F6P as well as on ATP, adenosine monophosphate (AMP), and most importantly its product FBP. The positive feedback of FBP onto PFK is responsible for glycolytic oscillations [37]. The PFK reaction rate is

$$
J_{\mathrm{PFK}}=v_{\mathrm{PFK}} \frac{w_{1110}+k_{\mathrm{PFK}} \sum_{i, j, l \in\{0,1\}} w_{i j 1 l}}{\sum_{i, j, k, l \in\{0,1\}} w_{i j k l}},
$$

with weights

$$
w_{i j k l}=\frac{\left(\mathrm{AMP} / K_{1}\right)^{i}\left(\mathrm{FBP} / K_{2}\right)^{j}\left(\mathrm{~F} 6 \mathrm{P} / K_{3}\right)^{k}\left(\mathrm{ATP} / K_{4}\right)^{l}}{f_{13}^{i k} f_{23}^{j k} f_{41}^{i l} f_{42}^{j l} f_{43}^{k l}},
$$

where $\mathrm{AMP}=\frac{\mathrm{ADP}^{2}}{\mathrm{ATP}}$. The $\mathrm{PDH}$ reaction rate also has a key feedback pathway, with facilitation of the reaction by mitochondrial $\mathrm{Ca}^{2+}$ as discussed in [5]. This reaction is described 
by

$$
J_{\mathrm{PDH}}=v_{\mathrm{PDH}} s_{\infty}\left(c_{\mathrm{m}}\right) \sqrt{\mathrm{FBP}}
$$

where $v_{\mathrm{PDH}}$ is one of the key parameters that we vary to explore the impact of changing the glycolytic efflux rate. The $\mathrm{Ca}^{2+}$ feedback is through the $s_{\infty}$ function,

$$
s_{\infty}\left(c_{\mathrm{m}}\right)=\frac{c_{\mathrm{m}}}{K_{\mathrm{PDH}}+c_{\mathrm{m}}}
$$

The details of mitochondrial metabolism are not included in this model. Instead, we use a phenomenological model, in which ATP production is driven by the output of the glycolytic subsystem, $J_{\mathrm{PDH}}$. The newly synthesized ATP comes at the expense of ADP, where the ADP concentration is described by

$$
\frac{d \mathrm{ADP}}{d t}=\frac{\left\{\mathrm{ATP}-\exp \left[\left(1+2.2 \frac{J_{\mathrm{PDH}}}{0.05+J_{\mathrm{PDH}}}\right)\left(1-\frac{c}{0.35}\right)\right] \mathrm{ADP}\right\}}{\tau_{a}}
$$

The dependence on the cytosolic $\mathrm{Ca}^{2+}$ concentration reflects the effect of $\mathrm{Ca}^{2+}$ flux across the mitochondrial inner member on the mitochondrial membrane potential [21]. It is assumed that the sum of the cytosolic nucleotides is conserved, so

$$
\mathrm{ATP}=\frac{1}{2}\left[A_{\mathrm{tot}}+\sqrt{-4 \mathrm{ADP}^{2}+\left(A_{\mathrm{tot}}-\mathrm{ADP}\right)^{2}}-\mathrm{ADP}\right]
$$

where $A_{\text {tot }}$ is the total nucleotide concentration. 


\section{Results}

\section{Slow $\mathrm{Ca}^{2+}$ oscillations can be produced by very different metabolic mechanisms}

The IOM can, for the appropriate parameter choices, produce slow oscillations in the intracellular $\mathrm{Ca}^{2+}$ concentration that resemble those often observed in $\mathrm{Ca}^{2+}$ measurements from pancreatic islets [45], and these slow oscillations are driven by two entirely different mechanisms. Figure 2 shows the time courses of $\mathrm{Ca}^{2+}$, ATP, and FBP for $v_{\mathrm{PDH}}=0.009 \mu \mathrm{M} / \mathrm{ms}$ (left column) and $v_{\mathrm{PDH}}=0.002 \mu \mathrm{M} / \mathrm{ms}$ (right column). The $\mathrm{Ca}^{2+}$ and ATP time series are remarkably similar, but the FBP time courses are quite different. For low $v_{\mathrm{PDH}}$ the FBP time course exhibits a sawtooth pattern (Figure $2 \mathrm{~F}$ ), whereas for larger $v_{\text {PDH }}$ there are pulses of FBP appearing from an almost-zero baseline (Figure 2E). This substantial difference in the FBP time course is indicative of the fact that there are distinct mechanisms driving the slow $\mathrm{Ca}^{2+}$ oscillations (see companion paper, [28], for a fast-slow analysis of the oscillation mechanisms).

Both types of oscillations have been observed experimentally, though in different preparations. Pulses of FBP were observed in measurements from muscle extracts [41], which contain the same isoform of the rhythmogenic enzyme phosphofructokinase (PFK-M) that is dominant in $\beta$-cells [44]. In contrast, sawtooth FBP oscillations were observed in islets, using a Förster Resonance Energy Transfer (FRET) biosensor [29, 31]. Thus, the PFK-M isoform, found in both muscle extracts and islet $\beta$-cells can contribute to metabolic oscillations resulting in either pulsatile or sawtooth FBP patterns, reflecting different oscillation mechanisms. 
A
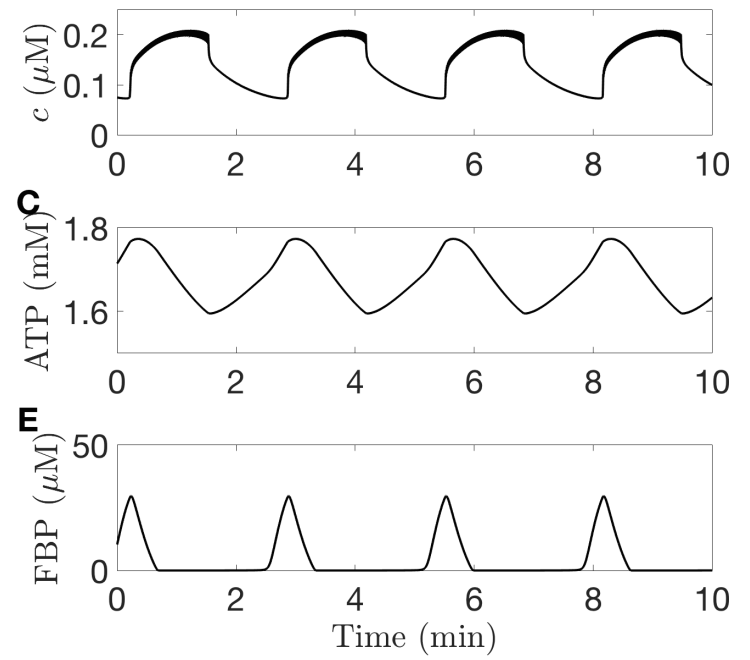

B

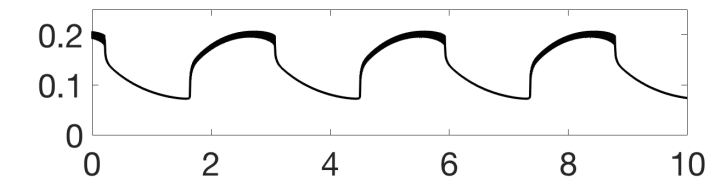

D

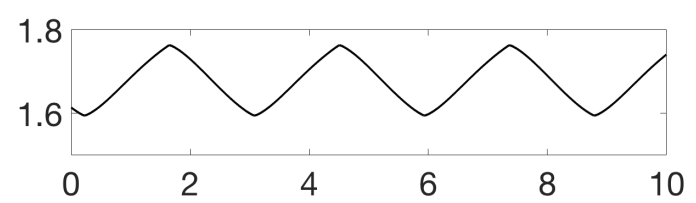

$\mathbf{F}$

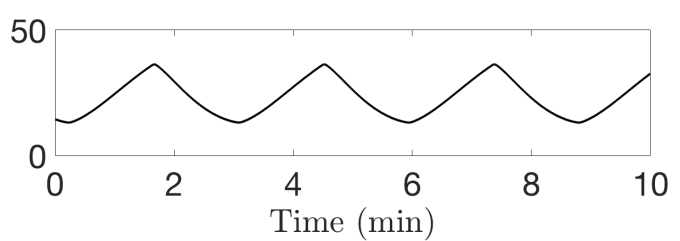

Figure 2: Time courses of cytosolic calcium (top row), cytosolic ATP (middle row), and FBP (bottom row), with $g_{\mathrm{K}(\mathrm{Ca})}=50 \mathrm{pS}$. In the left column $v_{\mathrm{PDH}}=0.009 \mu \mathrm{M} / \mathrm{ms}$; in the right column $v_{\mathrm{PDH}}=0.002 \mu \mathrm{M} / \mathrm{ms}$.

Our goals are to characterize conditions in which one can expect the different FBP patterns, and to determine the role played by the PFK enzyme in each.

\section{Pulsatile FBP occurs when the glycolytic efflux is sufficiently large}

We begin by exploring the $\left(g_{\mathrm{K}(\mathrm{Ca})}, v_{\mathrm{PDH}}\right)$-parameter space, where $g_{\mathrm{K}(\mathrm{Ca})}$ and $v_{\mathrm{PDH}}$ were chosen for the key roles they play in the electrical and metabolic components of the model. More precisely, variations in $g_{\mathrm{K}(\mathrm{Ca})}$ alter the active phase duration from tens of seconds (when $g_{\mathrm{K}(\mathrm{Ca})}$ is large) to several minutes (when $g_{\mathrm{K}(\mathrm{Ca})}$ is small). The mechanism for this effect was described using fast-slow analysis [6]. The parameter $v_{\mathrm{PDH}}$ controls the maximum efflux out of glycolysis. Thus, $v_{\mathrm{PDH}}$ determines the degree to which FBP can build up and feed back onto PFK in a regenerative manner. It is this positive feedback, which results in depletion 
of the substrate F6P, that is responsible for intrinsic PFK-induced oscillations [38]. The greater the value of $v_{\mathrm{PDH}}$ the less the positive feedback of FBP there will be onto PFK.

Figure 3 shows the results of running a grid of simulations over the range $g_{\mathrm{K}(\mathrm{Ca})} \in$ $[0,850] \mathrm{pS}$ and $v_{\mathrm{PDH}} \in[0,0.01] \mu \mathrm{M} / \mathrm{ms}$. We observe that FBP has a pulsatile time course for larger values of $v_{\mathrm{PDH}}$ and smaller values of $g_{\mathrm{K}(\mathrm{Ca})}$. The pulsatile and sawtooth regions of the parameter plane are separated by a bistable region (shaded, which we denote as BR), where FBP may have pulsatile or sawtooth behavior, depending on the initial conditions.

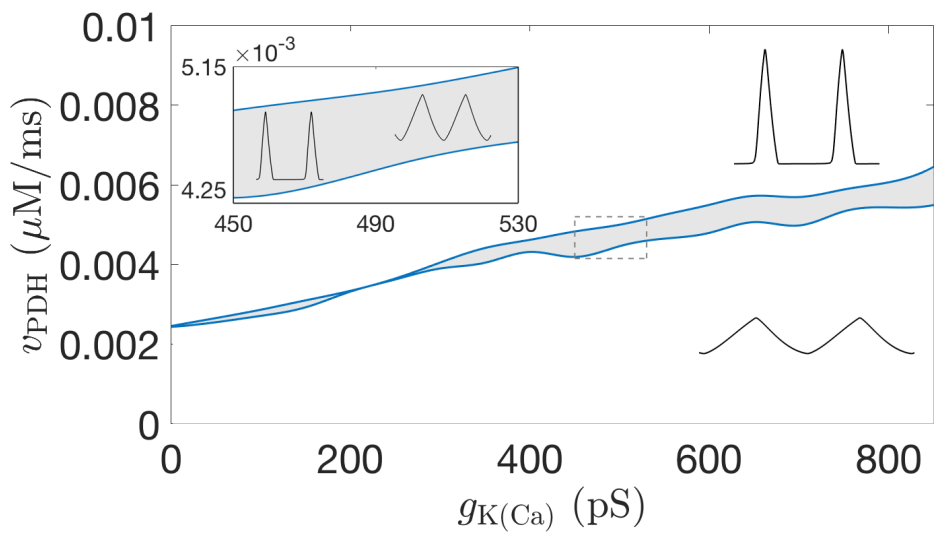

Figure 3: Partition of the $\left(g_{\mathrm{K}(\mathrm{Ca})}, v_{\mathrm{PDH}}\right)$ parameter plane into a region producing FBP pulses and a region producing sawtooth FBP time courses, separated by a bistable region (BR, shaded). Inset: zoom of the BR, where FBP can be either sawtooth or pulsatile, depending on the initial conditions. Here and elsewhere, pulsatile and sawtooth time courses are distinguished by eye.

\section{The relationship between oscillations in the glycolytic subsystem and the shape of the FBP time course}

What is the basis for the qualitatively different FBP time courses? What determines the boundary between sawtooth and pulsatile FBP time courses in Figure 3? The answers 
must lie within the glycolytic subsystem that produces intrinsic glycolytic oscillations, i.e., oscillations that persist when the variables in the electrical module are clamped. We note that the glycolytic subsystem (9) depends explicitly on $v_{\mathrm{PDH}}$ and implicitly on $g_{\mathrm{K}(\mathrm{Ca})}$. More precisely, $g_{\mathrm{K}(\mathrm{Ca})}$ plays a role in determining the intracellular $\mathrm{Ca}^{2+}$ level, $c$. For a fixed $c$, the equilibrium mitochondrial $\mathrm{Ca}^{2+}$ level is

$$
c_{\mathrm{m}}=\frac{\left(k_{\mathrm{uni}}+k_{\mathrm{NaCa}}\right) c}{k_{\mathrm{NaCa}}},
$$

which, in turn, affects the glycolytic efflux via the $\mathrm{PDH}$ reaction rate, $J_{\mathrm{PDH}}$, and the $\mathrm{Ca}^{2+}$ feedback via $s_{\infty}$ (see Eqs. 12,13). Cytosolic $\mathrm{Ca}^{2+}$ also affects the equilibrium ATP production through Eqs. 14 and 15. Finally, the nuclueotides AMP, ADP, and ATP influence PFK activity through Eqs. 10 and 11. Thus, $g_{\mathrm{K}(\mathrm{Ca})}$ affects glycolysis indirectly through its influence on $c$. In what follows, we isolate the glycolytic subsystem (9) by clamping $c$ at a fixed value and assuming ATP at the equilibrium, and perform bifurcation studies of the intrinsic oscillations with respect to $c$ and $v_{\mathrm{PDH}}$.

We first set $v_{\mathrm{PDH}}=0.009 \mu \mathrm{M} / \mathrm{ms}$ and construct a bifurcation diagram of the glycolytic subsystem with $c$ as a bifurcation parameter. Figure $4 \mathrm{~A}$ shows that for low values of $c$ the glycolytic subsystem is stationary. Lower values of $c$ produce higher equilibrium values of FBP, due to the influence of $c$ on $J_{\mathrm{PDH}}$; with lower values of $c$ the efflux through glycolysis is low, so FBP builds up. At $c \approx 0.04 \mu \mathrm{M}$ there is a subcritical Hopf bifurcation (HB, blue square), giving rise to a branch of unstable periodic solutions that meets a branch of stable periodic solutions at a saddle-node of periodics bifurcation (SNP, red diamonds). Thus, for $c$ values to the right of the SNP point, the attractor of the glycolytic subsystem is a limit 
A
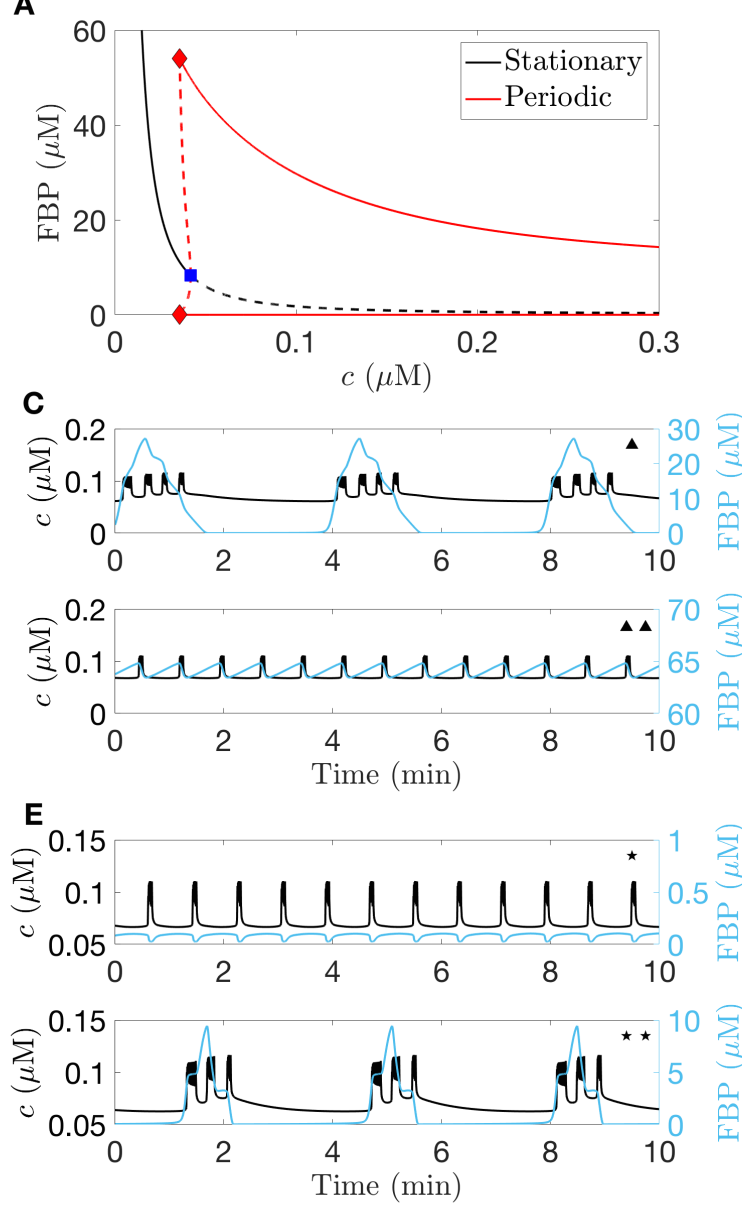

B
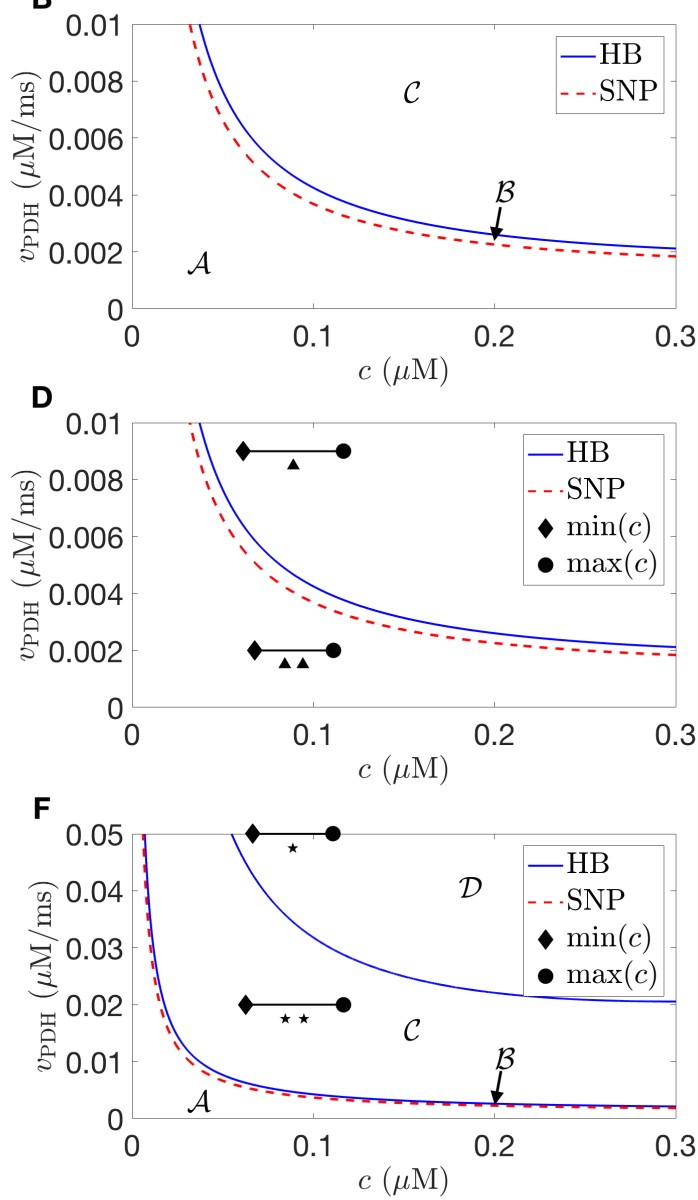

Figure 4: (A) Bifurcation structure of the glycolytic module with respect to $c$, for fixed $v_{\mathrm{PDH}}=0.009 \mu \mathrm{M} / \mathrm{ms}$. The blue and red squares indicate the subcritical HB and the SNP, respectively. (B) Two-parameter bifurcation diagram of the glycolytic subsystem showing the regions of $\mathrm{Ca}^{2+}$-dependent $(\mathcal{A})$, conditional $\mathrm{Ca}^{2+}$-independent $(\mathcal{B})$, and $\mathrm{Ca}^{2+}$-independent $(\mathcal{C})$ oscillations. (C) Calcium and FBP time courses with $g_{\mathrm{K}(\mathrm{Ca})}=800 \mathrm{pS}$, and $v_{\mathrm{PDH}}=0.009$ $\mu \mathrm{M} / \mathrm{ms}$ (top) and $v_{\mathrm{PDH}}=0.002 \mu \mathrm{M} / \mathrm{ms}$ (bottom). (D) $\mathrm{Ca}^{2+}$ oscillations from panel C (black segments) projected into the $\left(c, v_{\mathrm{PDH}}\right)$ plane. The triangles identify the corresponding time courses in the left panel. (E) $\mathrm{Ca}^{2+}$ and FBP time courses with $v_{\mathrm{PDH}}=0.05 \mu \mathrm{M} / \mathrm{ms}$ (top) and $v_{\mathrm{PDH}}=0.02 \mu \mathrm{M} / \mathrm{ms}$ (bottom) with $g_{\mathrm{K}(\mathrm{Ca})}=800 \mathrm{pS}$. (F) Projections of the $\mathrm{Ca}^{2+}$ oscillations (black segments) from panel A into the $\left(c, v_{\mathrm{PDH}}\right)$ plane. The stars identify the corresponding time courses in the left panel. 
cycle.

Figure 4B shows the two-parameter continuations of the Hopf and SNP bifurcations in the $\left(c, v_{\mathrm{PDH}}\right)$ plane. The HB and SNP curves partition the plane into three regions, each characterized by different dynamics: in region $\mathcal{A}$ (below the SNP curve) the equilibrium is a unique attractor; in region $\mathcal{C}$ (above the HB curve) the equilibrium is unstable and the limit cycle is the only attractor; in region $\mathcal{B}$ (between the two curves) both the steady state and the limit cycle are stable. This figure, then, characterizes the asymptotic structure of the glycolytic subsystem with the input variable $c$ clamped and the input nucleotide variables such as ATP constant at their corresponding equilibrium values. The oscillations present in region $\mathcal{C}$ are intrinsic to the glycolytic subsystem.

We return now to the full IOM, where $c$ and the nucleotides vary over time. The top panel of Figure $4 \mathrm{C}$ shows $c$ and FBP time courses for a $\left(g_{\mathrm{K}(\mathrm{Ca})}, v_{\mathrm{PDH}}\right)$ parameter combination that results in a pulsatile FBP pattern, while the bottom panel corresponds to a sawtooth FBP time course. In the first case, compound bursting is produced, consisting of fast bursts grouped into episodes that occur during each FBP pulse. Panel D shows, for each of these two cases, the range of $c$ values taken on during oscillations. From this panel we see that when FBP oscillations are sawtooth, the projection of the orbit lies in region $\mathcal{A}$ of the $\left(c, v_{\mathrm{PDH}}\right)$ plane, corresponding to the case where glycolysis is stationary. In the case of the pulsatile FBP time course in Figure $4 \mathrm{C}$ the projection into the $\left(c, v_{\mathrm{PDH}}\right)$ plane lies in region $\mathcal{C}$, where the glycolytic subsystem is oscillatory.

More generally, if the periodic orbit of the IOM lies in region $\mathcal{A}$ of Figure $4 \mathrm{~B}$ then FBP will have a sawtooth shape, and the oscillations would stop if $c$ were clamped. If the projection of the periodic orbit lies in region $\mathcal{C}$ then FBP will have a pulsatile shape due to 
the presence of intrinsic glycolytic oscillations that would persist even if $c$ were clamped. If it crosses through region $\mathcal{B}$ then the FBP time course could be sawtooth or pulsatile.

\section{Too much glycolytic flux destroys the intrinsic glycolytic oscillations}

Figure 4A-D shows that intrinsic glycolytic oscillations only occur if the $\mathrm{PDH}$ reaction rate is sufficiently large. If $v_{\mathrm{PDH}}$ is too small, then the FBP level will build up to such a high level that the PFK reaction will always stay elevated and there will be no glycolytic oscillations. But can $v_{\mathrm{PDH}}$ be too large to permit glycolytic oscillations? It seems reasonable that with very large efflux through glycolysis the FBP level should be too low to provide the necessary positive feedback onto PFK for glycolytic oscillations. This is in fact what happens. There is a second $\mathrm{HB}$ in the glycolytic subsystem, which occurs for large $v_{\mathrm{PDH}}$, and above which the glycolytic subsystem is stationary.

Figure $4 \mathrm{E}$ shows two examples of bursting oscillations produced by the IOM with $g_{\mathrm{K}(\mathrm{Ca})}=$ $800 \mathrm{pS}$ and both with large values of $v_{\mathrm{PDH}}$. In the bottom example $\left(v_{\mathrm{PDH}}=0.02 \mu \mathrm{M} / \mathrm{ms}\right)$ compound bursting is produced, but now the FBP pulses are much smaller than those produced during compound bursting in Figure $4 \mathrm{C}$ (with $v_{\mathrm{PDH}}=0.009 \mu \mathrm{M} / \mathrm{ms}$ ). The top panel uses an even larger maximum efflux value of $v_{\mathrm{PDH}}=0.05 \mu \mathrm{M} / \mathrm{ms}$, and now fast bursting is produced. In this case, there are no FBP pulses and FBP stays near zero since the glycolytic efflux rate is so high. When the projections of these orbits are plotted in the $\left(c, v_{\mathrm{PDH}}\right)$ plane (Figure $4 \mathrm{~F}$ ), the compound bursting rhythm lies in region $\mathcal{C}$ where there are intrinsic glycolytic oscillations. In contrast, the fast bursting projection lies in a new region of stationary attractors, region $\mathcal{D}$, which is separated from region $\mathcal{C}$ by the upper curve of Hopf bifurcations. 
In summary, the transition from fast bursting to compound bursting shown in Figure $4 \mathrm{C}$ when $v_{\mathrm{PDH}}$ was increased from $0.002 \mu \mathrm{M} / \mathrm{ms}$ to $0.009 \mu \mathrm{M} / \mathrm{ms}$ occurred because the lower threshold for intrinsic glycolytic oscillations was crossed, turning on the oscillations. The transition from compound bursting oscillations back to fast bursting when $v_{\mathrm{PDH}}$ was increased from $0.02 \mu \mathrm{M} / \mathrm{ms}$ to $0.05 \mu \mathrm{M} / \mathrm{ms}$ occurred because the upper threshold for intrinsic glycolytic oscillations was crossed, turning off the oscillations.

\section{Bursting patterns and their relationship to metabolic activity}

The parameter $g_{\mathrm{K}(\mathrm{Ca})}$ affects the cell's electrical activity directly through the ionic current $I_{\mathrm{K}(\mathrm{Ca})}(\mathrm{Eq} .4)$. The parameter $v_{\mathrm{PDH}}$ affects the electrical activity indirectly through its effects on ATP production and downstream inhibitory effects of ATP on the K(ATP) current (Eq. 5). We now determine how variations of these parameters affect the bursting patterns produced by the model cell. For the purposes of quantification, we use the following definitions. We say that the bursting rhythm is

- fast if the period is less than 2 min;

- slow if the period is greater than $2 \mathrm{~min}$;

- compound if the burst pattern consists of both long and short interburst intervals, and if the ratio of the longest to the shortest is greater than 1.25 .

The results of this classification are shown in the circle plot of Figure 5, where the color and size of the circles indicate the type and duration of the busting, respectively.

The first observation we make is that compound bursting only occurs between the lower and upper thresholds for pulsatile FBP oscillations. Thus, during compound oscillations the 


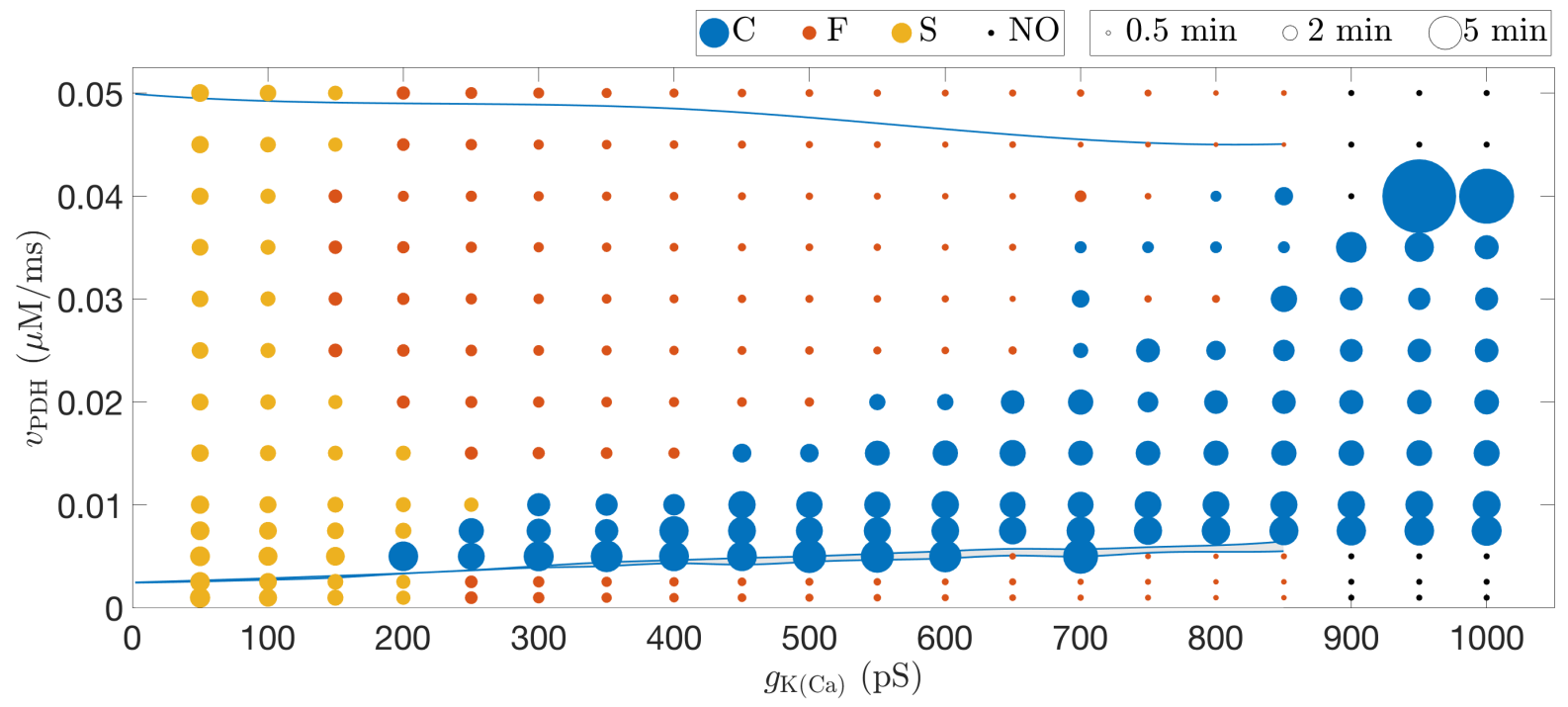

Figure 5: Classification of bursting oscillations with changes in $g_{\mathrm{K}(\mathrm{Ca})}$ and $v_{\mathrm{PDH}}$. The colors and radii of the circles identify the type of bursting and the burst duration, respectively. The upper and lower curves enclose the region of pulsatile FBP oscillations. C, F, S, and NO represent compound bursting, fast bursting, slow bursting, and no oscillations, respectively. The lower curves are the two curves shown in Figure 3.

FBP time course will be pulsatile. This prediction can be tested experimentally by simultaneously measuring either the membrane potential or $\mathrm{Ca}^{2+}$ (to see if compound oscillations are produced) together with a FRET indicator of the FBP concentration.

A second observation is that pulsatile FBP oscillations can occur coincident with either fast, slow, or compound bursting oscillations, since all three types occur between the lower and upper thresholds for pulsatile FBP. Both fast and slow bursting can also occur coincident with a sawtooth FBP time course (bottom and top regions of the figure). Thus, unless compound oscillations are produced, it is not possible to determine from the $\mathrm{Ca}^{2+}$ time course alone whether or not the glycolytic oscillator is active, as was seen in Figure 2.

A third observation from Figure 5 is that the burst period decreases with increases in 
$g_{\mathrm{K}(\mathrm{Ca})}$, up until the point at which compound oscillations are induced or the system becomes silent. In the former case, the glycolytic oscillations are sufficiently large that they begin to periodically initiate and terminate electrical bursting oscillations through their effect on the K(ATP) current. An analysis of compound bursting and the contribution of $\mathrm{Ca}^{2+}$ pulses to the glycolytic oscillations that underlies it was performed previously [43]. Once the bursting is converted to compound bursting the oscillation period increases dramatically as the compound burst period matches the period of the intrinsic glycolytic oscillator. For large or small values of $v_{\mathrm{PDH}}$ there are no glycolytic oscillations, and increasing $g_{\mathrm{K}(\mathrm{Ca})}$ eventually terminates all electrical activity and the model cell comes to rest.

Finally, we point out the large-period oscillations that occur at $g_{\mathrm{K}(\mathrm{Ca})}=950 \mathrm{pS}$ and $1000 \mathrm{pS}$, with $v_{\mathrm{PDH}}=0.04 \mu \mathrm{M} / \mathrm{ms}$. For these parameter combinations the silent phase between burst episodes becomes extremely long, suggesting that the system is close to a homoclinic bifurcation. The precise nature of this bifurcation is an open question. Indeed, for larger values of $v_{\mathrm{PDH}}$, the system is at rest with no electrical or metabolic oscillations.

Figure 6 shows $c$ and FBP time courses for a range of values of $g_{\mathrm{K}(\mathrm{Ca})}$ and $v_{\mathrm{PDH}}$, complementing the quantification of Figure 5. The value of $v_{\mathrm{PDH}}$ is the same along elements of each row of the figure, while $g_{\mathrm{K}(\mathrm{Ca})}$ increases from left to right. Panel A corresponds to a point in the upper left of Figure 5. Since this is above the upper threshold for pulsatile FBP, the FBP time course exhibits a sawtooth pattern. The FBP values are very low, however, since the glycolytic efflux is very large $\left(v_{\mathrm{PDH}}=0.05 \mu \mathrm{M} / \mathrm{ms}\right)$. The bursting is slow because $g_{\mathrm{K}(\mathrm{Ca})}$ has a small value. When $g_{\mathrm{K}(\mathrm{Ca})}$ is increased from $50 \mathrm{pS}$ to $600 \mathrm{pS}$ (panel B) the burst period decreases dramatically, but the FBP time course retains the sawtooth pattern. At $g_{\mathrm{K}(\mathrm{Ca})}=900 \mathrm{pS}$ all electrical activity stops since the $\mathrm{K}^{+}$current is so large that electrical 
impulses are not triggered.
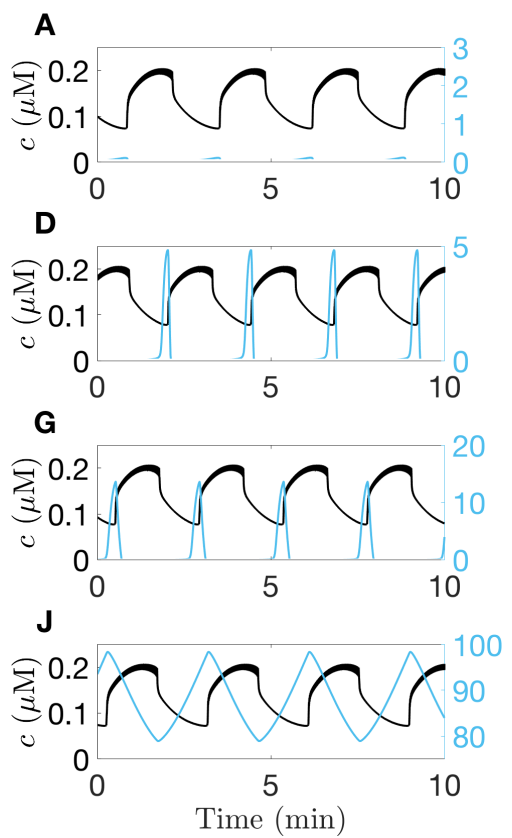

B
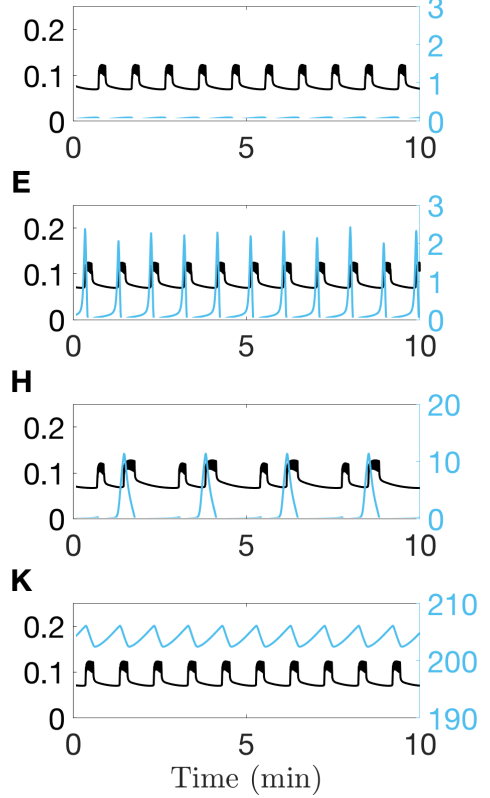

C
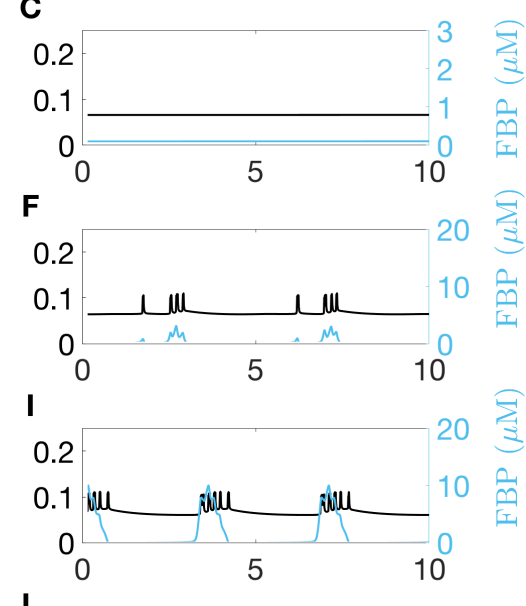

$\mathbf{L}$

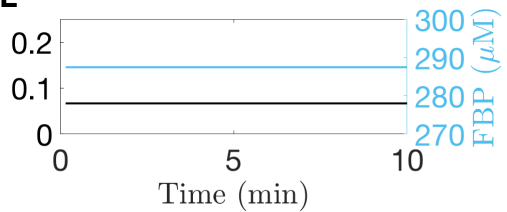

Figure 6: Calcium and FBP time courses taken over a grid in $g_{\mathrm{K}(\mathrm{Ca})}$ and $v_{\mathrm{PDH}}$. The sampled values are: $v_{\mathrm{PDH}}=0.05 \mu \mathrm{M} / \mathrm{ms}$ (top row), $v_{\mathrm{PDH}}=0.035 \mu \mathrm{M} / \mathrm{ms}$ (second row), $v_{\mathrm{PDH}}=$ $0.02 \mu \mathrm{M} / \mathrm{ms}$ (third row), $v_{\mathrm{PDH}}=0.001 \mu \mathrm{M} / \mathrm{ms}$ (bottom row); $g_{\mathrm{K}(\mathrm{Ca})}=50 \mathrm{pS}$ (left column), $g_{\mathrm{K}(\mathrm{Ca})}=600 \mathrm{pS}$ (middle column), $g_{\mathrm{K}(\mathrm{Ca})}=900 \mathrm{pS}$ (right column).

The second row of Figure 6 has less glycolytic efflux, with $v_{\mathrm{PDH}}$ reduced to $0.035 \mu \mathrm{M} / \mathrm{ms}$. This is below the upper threshold for pulsatile FBP oscillations, so the bursting along this row is coincident with FBP pulses. At the smallest value of $g_{\mathrm{K}(\mathrm{Ca})}$ shown oscillations are slow, but are now organized by the intrinsic glycolytic oscillator (in contrast to panel A). Increasing $g_{\mathrm{K}(\mathrm{Ca})}$ again speeds up the oscillations (panel E), but now at $g_{\mathrm{K}(\mathrm{Ca})}=900 \mathrm{pS}$ compound bursting is produced and the period increases significantly (panel F). In contrast, panel $\mathrm{C}$ shows that for the same value of $g_{\mathrm{K}(\mathrm{Ca})}$ (but a larger value of $v_{\mathrm{PDH}}$ ), there is no electrical activity at all. 
The third row of Figure 6 has even less glycolytic efflux $\left(v_{\mathrm{PDH}}=0.02 \mu \mathrm{M} / \mathrm{ms}\right)$, and as a result the FBP pulses are much larger than in the second row. Interestingly, the slow bursts in panel $\mathrm{G}$ seem to split into two shorter bursts in panel $\mathrm{H}$, creating compound oscillations. As $g_{\mathrm{K}(\mathrm{Ca})}$ is further increased the number of oscillations per episode increases, and the duration of each active phase decreases (panel I).

In the last row of Figure 6 the glycolytic efflux rate is sufficiently low that the system is below the lower threshold for FBP pulses and intrinsic glycolytic oscillations. Moreover, the low glycolytic efflux means that the FBP levels are also much higher than in the other

panels of Figure 6. Panel J shows slow bursting with a low $g_{\mathrm{K}(\mathrm{Ca})}$ that becomes much faster when $g_{\mathrm{K}(\mathrm{Ca})}$ is increased to $600 \mathrm{pS}$ (panel K). At the largest $g_{\mathrm{K}(\mathrm{Ca})}$ value shown all electrical activity stops (panel L), just as in panel $\mathrm{C}$ which was also outside the window for intrinsic glycolytic oscillations. We note that the mean FBP level increases with $g_{\mathrm{K}(\mathrm{Ca})}$ along this row, even though $v_{\mathrm{PDH}}$ is fixed. This is because the mean $\mathrm{Ca}^{2+}$ level is lower at higher values of $g_{\mathrm{K}(\mathrm{Ca})}$, and the lower $\mathrm{Ca}^{2+}$ results in less glycolytic efflux, causing FBP to increase.

\section{Diazoxide and $\mathrm{KCl}$ as tools for studying islet oscillations}

We showed in Figure 2 that slow $\mathrm{Ca}^{2+}$ oscillations, and the accompanying ATP oscillations, can look quite similar regardless of whether the glycolytic oscillator is on or off. Is the shape of the FBP timecourse the only way to determine whether the glycolytic oscillator is activated? Indeed, there is another way to determine this.

The protocol consists of two steps.

1. We first add the pharmacological agent diazoxide (Dz) to an islet in stimulatory glucose. 
This agent activates K(ATP) channels in $\beta$-cells, thus increasing the K(ATP) current and terminating all spiking activity. We simulated the application of $\mathrm{Dz}$ at $t=10 \mathrm{~min}$ by increasing $g_{\mathrm{K}(\mathrm{ATP})}$ from $25,000 \mathrm{pS}$ to $37,000 \mathrm{pS}$.

2. In the second step, we induce cell depolarization by adding $\mathrm{KCl}$, which increases the $\mathrm{K}^{+}$ Nernst potential and elevates the calcium concentration. We simulated the addition of $\mathrm{KCl}$ at $t=40 \mathrm{~min}$ by increasing $V_{K}$ from $-75 \mathrm{mV}$ to $-60 \mathrm{mV}$.

The results of this protocol are shown in Figure 7 for $v_{\mathrm{PDH}}=0.002 \mu \mathrm{M} / \mathrm{ms}$ (panel A), $v_{\mathrm{PDH}}=0.009 \mu \mathrm{M} / \mathrm{ms}$ (panel B), $v_{\mathrm{PDH}}=0.015 \mu \mathrm{M} / \mathrm{ms}$ (panel C), and $v_{\mathrm{PDH}}=0.05 \mu \mathrm{M} / \mathrm{ms}$ (panel D).

For $v_{\mathrm{PDH}}=0.002 \mu \mathrm{M} / \mathrm{ms}$ (Figure 7A) the glycolytic oscillator is not initially engaged, so the FBP oscillations have a sawtooth shape. When Dz is applied the electrical bursting and large $\mathrm{Ca}^{2+}$ oscillations stop, and so do the FBP oscillations. The FBP now increases to a plateau. When $\mathrm{KCl}$ is added, the $\mathrm{Ca}^{2+}$ concentration increases and the FBP level crashes. The rise of FBP with Dz application reflects the fact that the decline in $c$ shuts off most glycolytic flux through its action on the PDH enzyme, so FBP builds up. When $c$ is elevated with $\mathrm{KCl}$ it increases flux through glycolysis, so that the FBP level rapidly declines. This simulation can be thought of in terms of Figure 4B, D, F. Prior to the application of Dz the system moves within region $\mathcal{A}$, below the curve of Hopf bifurcations. Addition of Dz sops the $\mathrm{Ca}^{2+}$ oscillations, and with the now constant $c$ the system still lies within this region. The addition of $\mathrm{KCl}$ increases $c$, but the system remains in region $\mathcal{A}$.

In the second panel of the figure the same procedure is used, only this time with a larger value of the glycolytic flux $\left(v_{\mathrm{PDH}}=0.009 \mu \mathrm{M} / \mathrm{ms}\right)$. The model cell is initially bursting 
with pulsatile glycolytic oscillations. When Dz is applied the electrical bursting again stops, along with the metabolic oscillations (after transient damped oscillations). The metabolic oscillations can be rescued, however, by increasing the $\mathrm{Ca}^{2+}$ concentration with $\mathrm{KCl}$. In terms of Figure 4B, D, F, the system initially moved within region $\mathcal{C}$, but with $\mathrm{Dz}$ application it fell into region $\mathcal{A}$, where there are no glycolytic oscillations. The addition of $\mathrm{KCl}$ increases $c$ so that the system again lies within region $\mathcal{C}$, thus restarting the glycolytic oscillator.

For $v_{\mathrm{PDH}}=0.015 \mu \mathrm{M} / \mathrm{ms}$ (Figure $7 \mathrm{C}$ ), the FBP oscillations persist even though the electrical bursting and large $\mathrm{Ca}^{2+}$ oscillations have stopped. Instead, there are small subthreshold $\mathrm{Ca}^{2+}$ oscillations (see inset), reflecting small fluctuations in the cell's membrane potential due to the continuing metabolic oscillations (the oscillatory ATP acts on K(ATP) channels). When the cell is depolarized at $t=40 \mathrm{~min}$ by the simulated application of $\mathrm{KCl}$, the $\mathrm{Ca}^{2+}$ concentration is also increased and subthreshold oscillations persist, as do FBP oscillations. Prior to the application of $\mathrm{Dz}$ the system moves within region $\mathcal{C}$. Upon application of $\mathrm{Dz}$ the fluctuations in $c$ are much smaller, and the mean continues to lie in region $\mathcal{C}$. When the addition of $\mathrm{KCl}$ is simulated, the system moves rightward in the diagram, again staying within region $\mathcal{C}$. Thus, under all conditions the glycolytic subsystem is in an oscillatory state.

The final panel, with $v_{\mathrm{PDH}}=0.05 \mu \mathrm{M} / \mathrm{ms}$ (Figure 7D), is the opposite of the second panel. Here, the glycolytic oscillations are initially small and have a sawtooth shape, since the system lies in region $\mathcal{D}$ of Figure 4B, D, F. Application of Dz stops the bursting and large $\mathrm{Ca}^{2+}$ oscillations, but activates the glycolytic oscillator, so there are now pulsatile FBP oscillations. This occurs because the reduction in $c$ moved the system into region $\mathcal{C}$ where the glycolytic oscillator is active. When application of $\mathrm{KCl}$ is simulated the elevation in $c$ 
terminates the FBP oscillations, since the system now once again lies in region $\mathcal{D}$.

Thus, the model predicts that when bursting oscillations are driven by an intrinsic glycolytic oscillator, metabolic oscillations may or may not persist with the application of Dz, and thus subthreshold $\mathrm{Ca}^{2+}$ oscillations may or may not be produced. However, if metabolic oscillations are terminated, they can be rescued with cell depolarization via $\mathrm{KCl}$. In contrast, in conditions where bursting is not driven by an intrinsic glycolytic oscillator, the application of Dz may actually activate the glycolytic oscillator, but depolarization by the application of $\mathrm{KCl}$ will inactive it. Thus, application of $\mathrm{KCl}$ facilitates metabolic oscillations (in the presence of Dz) when the cell initially has an active glycolytic oscillator, and it suppresses metabolic oscillations when the glycolytic oscillator is not initially activated.

\section{Slow $\mathrm{Ca}^{2+}$ oscillations can be produced with different isoforms of PFK}

The muscle isoform of the allosteric enzyme PFK, called PFK-M, is capable of producing glycolytic oscillations. This was demonstrated in muscle extracts [42], and is the basis for the intrinsic glycolytic oscillations produced by our model that lead to pulsatile FBP. However, two other isoforms of PFK are expressed in $\beta$-cells: PFK-C is prominent in the brain, while PFK-L is prominent in the liver. These two isoforms of PFK have a lower affinity for FBP feedback [8], and a higher affinity for ATP [16, 17]. Although all three isoforms are expressed in $\beta$-cells, the isoform responsible for most of the PFK activity is PFK-M [44].

What if the fraction of M-type PFK were reduced, what effect would that have on the parameter window for intrinsic glycolytic oscillations? We explored this in an earlier 


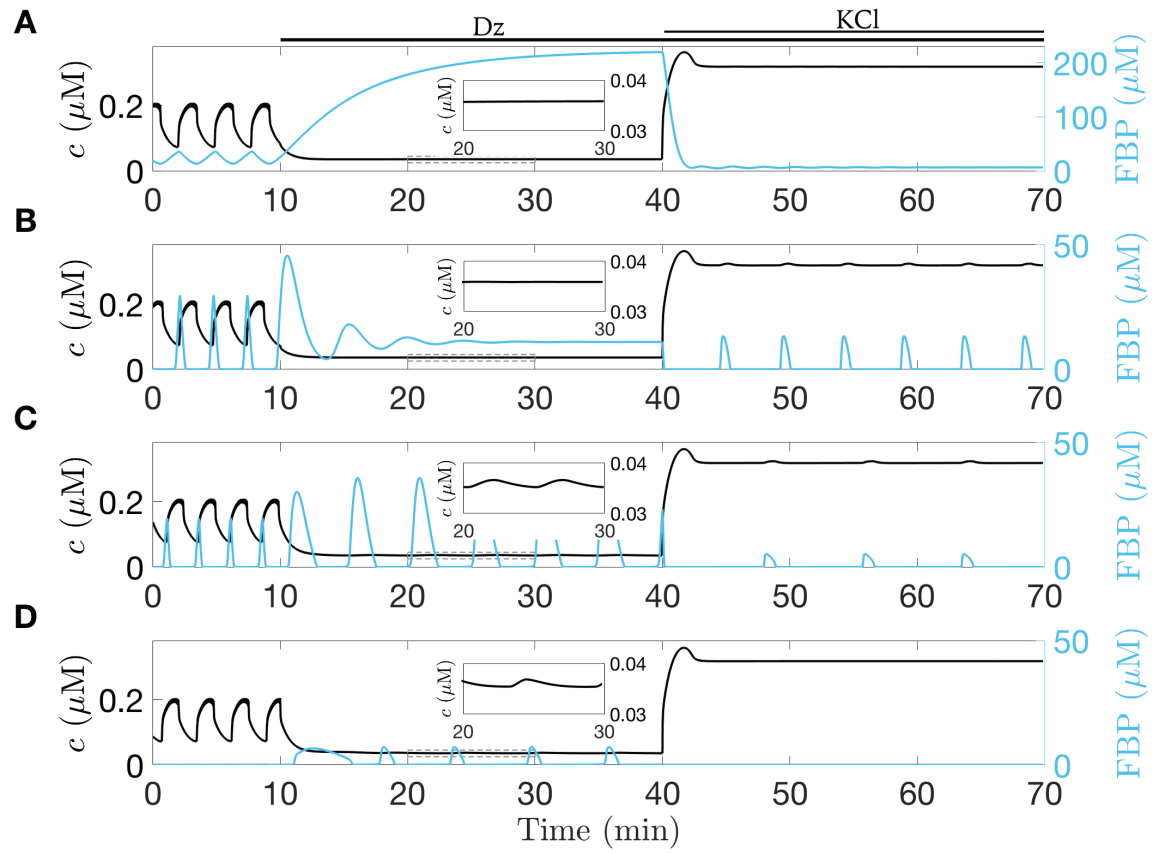

Figure 7: Two step protocol to determine whether the glycolytic oscillator is active. (A) For $v_{\mathrm{PDH}}=0.002 \mu \mathrm{M} / \mathrm{ms}$, metabolic oscillations require $\mathrm{Ca}^{2+}$ oscillations, and so are terminated by the application of the K(ATP) channel opener Dz (simulated by increasing $g_{\mathrm{K}(\mathrm{ATP})}$ from $25,000 \mathrm{pS}$ to $37,000 \mathrm{pS}$ ) and rescue of oscillations by $\mathrm{KCl}$ (simulated by increasing $V_{K}$ from $-75 \mathrm{mV}$ to $-60 \mathrm{mV}$ ) is imposssible. (B) For $v_{\mathrm{PDH}}=0.009 \mu \mathrm{M} / \mathrm{ms}$, metabolic oscillations are terminated by $\mathrm{Dz}$, but can be rescued with the application of $\mathrm{KCl}$. (C) For $v_{\mathrm{PDH}}=0.015$ $\mu \mathrm{M} / \mathrm{ms}$, metabolic oscillations and subthreshold $\mathrm{Ca}^{2+}$ oscillations persist after the application of $\mathrm{Dz}$ and subsequent application of $\mathrm{KCl}$. (D) For $v_{\mathrm{PDH}}=0.05 \mu \mathrm{M} / \mathrm{ms}$, application of Dz produces pulsatile FBP oscillations and subthreshold $\mathrm{Ca}^{2+}$ oscillations. These are terminated by application of $\mathrm{KCl}$. Note that the FBP scale is different between the first panel and subsequent panels. In all cases, $g_{\mathrm{K}(\mathrm{Ca})}=50 \mathrm{pS}$.

publication, but in the model used for that study there was no $\mathrm{Ca}^{2+}$ feedback onto glycolysis [4]. We therefore re-examine the question here, noting that up to this point the assumption has been that all of the PFK is M-type. We now consider the possibility that some fraction, $M_{\text {frac }} \in[0,1]$, of the total PFK is of M-type and the remainder is of C-type. The total PFK 
flux then, is

$$
J_{\mathrm{PFK}}=M_{\mathrm{frac}} J_{\mathrm{PFK}-\mathrm{M}}+\left(1-M_{\mathrm{frac}}\right) J_{\mathrm{PFK}-\mathrm{C}},
$$

where $J_{\mathrm{PFK}-\mathrm{M}}$ and $J_{\mathrm{PFK}-\mathrm{C}}$ are the portion of flux due to the M- and C-type isoforms, respectively. The two fluxes, $J_{\mathrm{PFK}-\mathrm{M}}$ and $J_{\mathrm{PFK}-\mathrm{C}}$, are defined as in (10) but with different values for the parameters $K_{2}$ and $K_{4}$. The C-type isoform has a lower affinity for FBP, so $K_{2}=2 \mu \mathrm{M}$ for $J_{\mathrm{PFK}-\mathrm{C}}$ rather than $1 \mu \mathrm{M}$, and a higher affinity for ATP, so $K_{4}=100 \mu \mathrm{M}$ rather than $1000 \mu \mathrm{M}$.

Figure 8A shows two examples of slow $\mathrm{Ca}^{2+}$ oscillations. In each case the oscillations are produced in spite of replacement of some M-type PFK with C-type. In the top example, only $40 \%$ of the PFK is M-type, while in the bottom example none of the PFK is of Mtype. Clearly, in this model slow $\mathrm{Ca}^{2+}$ oscillations do not require M-type PFK for their production. Note, however, that in both cases the FBP oscillations have a sawtooth form since the glycolytic oscillator is not active.

Replacing some fraction of PFK-M with PFK-C causes a change in the window for intrinsic glycolytic oscillations. To see this, we return to the glycolytic subsystem, treating $c$ as a parameter as in Figure 4D, F. In Figure 8B we show the upper and lower threshold curves for glycolytic oscillations for five values of $M_{\text {frac }}$. As the fraction of M-type PFK is reduced, the upper threshold curve moves downward, reducing the size of the window for intrinsic glycolytic oscillations. By $M_{\text {frac }}=0.35$ the upper and lower threshold curves have coalesced, and as $M_{\text {frac }}$ is decreased further the region of intrinsic glycolytic oscillations decreases in size and becomes restricted to larger values of the cytosolic $\mathrm{Ca}^{2+}$ concentration. Thus, the likelihood that slow $\mathrm{Ca}^{2+}$ oscillations are driven by intrinsic glycolytic oscillations 
A

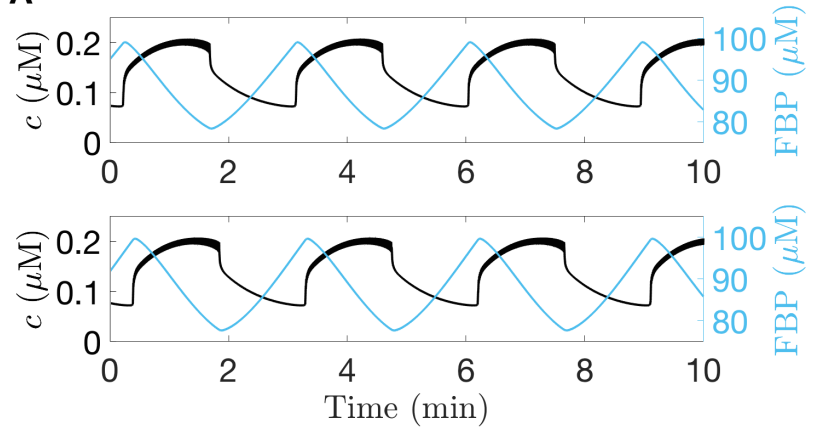

B

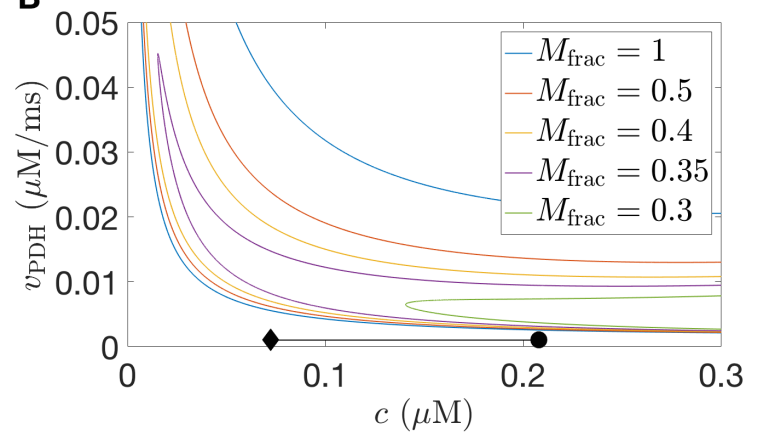

Figure 8: (A) $\mathrm{Ca}^{2+}$ and FBP time courses for $g_{\mathrm{K}(\mathrm{Ca})}=50 \mathrm{pS}, v_{\mathrm{PDH}}=0.001 \mu \mathrm{M} / \mathrm{ms}$, and $M_{\text {frac }}=0.4$ (top) and $M_{\text {frac }}=0$ (bottom). (B) Two-parameter bifurcation structure of the glycolytic subsystem showing the Hopf bifurcation curves for various values of $M_{\text {frac }}$, together with the projections of the $\mathrm{Ca}^{2+}$ oscillations from panel A (black segment).

in the full IOM declines as PFK-M is replaced by PFK-C. Moreover, if the percentage of M-type PFK falls below about $25 \%$ it becomes very unlikely or impossible for this to occur.

Figure 8 demonstrates first that slow $\mathrm{Ca}^{2+}$ oscillations should be possible in islets in which most or all of the M-type PFK is replaced with another isoform. This prediction was made recently with a somewhat different $\beta$-cell model [27]. The figure also demonstrates that slow $\mathrm{Ca}^{2+}$ oscillations that are produced in $\beta$-cells under this condition should be coincident with a sawtooth FBP time course since there will be no intrinsic glycolytic oscillations. In fact, data have shown that slow $\mathrm{Ca}^{2+}$ oscillations can occur in islets in which the PFK-M protein has been genetically knocked out [34], where it is expected that other PFK isoforms will be upregulated. It has not been determined whether FBP oscillates in these islets and, if so, whether the timecourse has a sawtooth time course. 


\section{Discussion}

The $\beta$-cells of pancreatic islets exhibit a wide range of oscillatory behavior, as observed in voltage traces, $\mathrm{Ca}^{2+}$ imaging, oxygen utilization, $\mathrm{NADH}$, and mitochondrial membrane potential time courses [7]. These data motivated the development of the Dual Oscillator Model [3], in which oscillations in glycolysis drive the electrical and $\mathrm{Ca}^{2+}$ oscillations of the cells. Data published later showing a sawtooth time course of the glycolytic metabolite FBP [29, 31] forced a re-thinking of the oscillation mechanism, resulting in the development of the Integrated Oscillator Model in which $\mathrm{Ca}^{2+}$ feedback onto glycolysis is the driving force behind the oscillations [27], and which is analyzed in the companion paper [28]. In the current paper using the IOM, we investigated how transitions can be made between the two types of oscillations. Since the model reflects the interaction of electrical and metabolic subsystems, bifurcation parameters from these two subsystems were used in the analysis.

We found that the transitions made between the two modes of oscillation in the full model can largely be understood from the glycolytic subsystem alone. In particular, there are lower and upper thresholds for intrinsic glycolytic oscillations. Between the thresholds, glycolytic oscillations are produced and can persist with the $\mathrm{Ca}^{2+}$ clamped, and these yield pulses of FBP. Outside of the thresholds any oscillations in metabolism that occur are due to $\mathrm{Ca}^{2+}$ feedback and would stop immediately if $\mathrm{Ca}^{2+}$ is clamped. The FBP time course in this case has a sawtooth shape, as reported in experiments [29, 31].

While this result, and the match to experimental data, may suggest that metabolic oscillations and the accompanying electrical and $\mathrm{Ca}^{2+}$ oscillations in islet $\beta$-cells are due to $\mathrm{Ca}^{2+}$ feedback, there are caveats. First, the data published thus far only show FBP 
oscillations when the islets exhibit slow bursting. No data have been published showing FBP oscillations during compound bursting, which is a prominent behavior in islets. One of the main results of this report is that, in the IOM, compound bursting is always due to intrinsic glycolytic oscillations. (We have shown previously that compound bursting can be produced by a model without glycolytic oscillations [2], but this was in a limited parameter range and there is no experimental evidence that this alternate mechanism occurs in $\beta$-cells.) Thus, we predict that if FBP were measured in a compound bursting islet, then the FBP time course would be pulsatile, not sawtooth.

Another caveat to the proposition that metabolic oscillations are always driven by $\mathrm{Ca}^{2+}$ feedback are data showing oscillations when the $\mathrm{Ca}^{2+}$ level is not oscillating. These data include the observation of oscillations in the number of $\mathrm{K}(\mathrm{ATP})$ channel openings in $\beta$-cells bathed in a low, sub-stimulatory, concentration of glucose [14]. A later study focused again on $\mathrm{K}(\mathrm{ATP})$ channel activity, but this time measuring $\mathrm{K}(\mathrm{ATP})$ channel conductance in a $\beta$-cell from an intact islet bathed in a stimulatory level of glucose. In both cases, the cellattached configuration of the patch clamp technique was used, which clamps the membrane potential and thereby also clamps the $\mathrm{Ca}^{2+}$ level in the cell.

Subthreshold $\mathrm{Ca}^{2+}$ oscillations have been measured in islets exposed to a low glucose level [7]. This preparation is similar to exposure of islets to a stimulatory glucose concentration along with the K(ATP) channel activator diazoxide, in that lowering the glucose level opens K(ATP) channels, just as diazoxide does. In another experiment, diazoxide was added to islets in stimulatory glucose just as in our simulations, and the autofluorescence of the adenine dinucleotide $\mathrm{NAD}(\mathrm{P}) \mathrm{H}$ was measured as a readout of metabolism [30]. It was found that in some cases the metabolic oscillations persisted during the application of diazoxide, 
as in Figure 7C. In some cases where metabolic oscillations did not persist, they could be rescued by depolarizing the islet with $\mathrm{KCl}$, as in Figure 7B. Finally, in some cases the $\mathrm{NAD}(\mathrm{P}) \mathrm{H}$ oscillations were not rescued by $\mathrm{KCl}$, as in Figure 7A, D. In a later study [31], the fluorescent sensor PKAR was used to detect the level of FBP. It was found that the PKAR signal initially had a sawtooth pattern, and upon application of Dz it stopped oscillating and increased significantly. When $\mathrm{KCl}$ was applied later the PKAR level crashed. Our model (Figure 7A) resembles this experimental behavior.

The experimental data therefore support a model in which metabolic oscillations in islets are sometimes driven by $\mathrm{Ca}^{2+}$ feedback and sometimes due to an intrinsic glycolytic oscillator. We have demonstrated that the oscillation mechanism depends on parameter values in both the electrical and metabolic subsystems, and manipulations such as changes in the glucose level or the clamping of $\mathrm{Ca}^{2+}$ can move the system from one oscillatory regime to the other. In fact, oscillations can persist even after the partial or complete replacement of the rhythmogenic enzyme PFK-M with a non-rhythmogenic isoform (Figure 8).

The first model for electrical bursting in $\beta$-cells was very simple, with oscillations driven by $\mathrm{Ca}^{2+}$ feedback onto $\mathrm{Ca}^{2+}$-activated $\mathrm{K}^{+}$channels [11]. Although it was the foundation for all subsequent $\beta$-cell models, the model provided a burst mechanism that could account for only one form of bursting, and was not particularly robust to parameter changes. The IOM is much more complex and one may ask why this complexity is needed. We believe that the answer is now apparent: the payoff for this complexity is a much greater range of potential activity patterns, and a much greater robustness in the generation of slow or compound oscillations. Indeed, mouse islets produce slow oscillations in activity under various physiological conditions, and even following genetic modification to knock out K(ATP) channels 
$[15,36]$ or the PFK-M isoform [34]. This robustness is perhaps a safeguard to ensure that insulin secretion is pulsatile, which is more effective at lowering the blood glucose level than constant insulin secretion $[9,25]$.

\section{Acknowledgements}

Thanks to the Department of Mathematics and the Institute of Molecular Biophysics at Florida State University for hosting I. Marinelli's internship. R. Bertram was supported by grant number DMS-1612193 from the National Science Foundation. I. Marinelli and L. Gerardo-Giorda were supported by the Basque Government through the BERC 20142017 program, and by Spanish Ministry of Economy and Competitiveness MINECO: BCAM Severo Ochoa excellence accreditation SEV-2013- 0323 and the Spanish "Plan Estatal de Investigacion, Desarrollo e Innovacion Orientada a los Retos de la Sociedad" under grant BELEMET - Brain ELEctro-METabolic modeling and numerical approximation (MTM2015-

69992-R). L. Gerardo-Giorda was also supported by grant BG2016 (KK-2016/00026) from Basque Government program ELKARTEK.

\section{References}

[1] A. Arredounani, J.-C. Henquin, And P. Gilon, Contribution of the endoplasmic reticulum to the glucose-induced $\left[\mathrm{Ca}^{2+}\right]_{\mathrm{c}}$ response in mouse pancreatic islets, Am. J. Physiol., 1330 (2002), pp. E982-E991. 
[2] R. Bertram, J. Rhohds, and W. P. Cimbora, A phantom bursting mechanism for episodic bursting, Bull. Math. Biol., 70 (2008), pp. 1979-1993.

[3] R. Bertram, L. Satin, M. Zhang, P. Smolen, and A. Sherman, Calcium and glycolysis mediate multiple bursting modes in pancreatic islets, Biophys. J., 87 (2004), pp. $3074-3087$.

[4] R. Bertram, L. S. Satin, M. G. Pedersen, D. S. Luciani, and A. SherMAN, Interaction of glycolysis and mitochondrial respiration in metabolic oscillations of pancreatic islets, Biophys. J., 92 (2007), pp. 1544-1555.

[5] R. Bertram, L. S. Satin, and A. Sherman, Closing in on the mechanisms of pulsatile insulin secretion, Diabetes, 67 (2018), pp. 351-359.

[6] R. Bertram and A. Sherman, A calcium-based phantom bursting model for pancreatic islets, Bull. Math. Biol., 66 (2004), pp. 1313-1344.

[7] R. Bertram, A. Sherman, and L. S. Satin, Metabolic and electrical oscillations: Partners in controlling pulsatile insulin secretion, Am. J. Physiol., 293 (2007), pp. E890E900.

[8] L. Bosca, J. J. Aragon, And A. Sols, Specific activation by fructose 2,6bisphosphate and inhibition by p-enolpyruvate of ascites tumor phosphofructokinase, Biochem. Biophys. Res. Commun., 106 (1982), pp. 486-491.

[9] P. R. Bratusch-Marrain, M. Komjati, and W. K. Waldhüusl, Efficacy of pulsatile versus continuous insulin administration on hepatic glucose production and glucose utilization in type I diabetic humans, Diabetes, 35 (1986), pp. 922-926. 
[10] C. Y. Cha, Y. Nakamura, Y. Himeno, J. Wang, S. Fujimoto, N. Inagaki, Y. E. EARm, AND A. NomA, Ionic mechanisms and $\mathrm{Ca}^{2+}$ dynamics underlying the glucose response of pancreatic $\beta$ cells, Am. J. Physiol., 138 (2011), pp. 21-37.

[11] T. R. Chay And J. KeIZer, Minimal model for membrane oscillations in the pancreatic $\beta$-cell, Biophys. J., 42 (1983), pp. 181-190.

[12] D. L. Cook, Isolated islets of Langerhans have slow oscillations of electrical activity, Metabolism, 32 (1983), pp. 681-685.

[13] F. Diederichs, Mathematical simulation of membrane processes and metabolic fluxes of the pancreatic $\beta$-cell, Bull. Math. Biol., 68 (2006), pp. 1779-1818.

[14] S. Dryselius, P.-E. Lund, E. Glyfe, And B. Hellman, Variations in ATPsensitive $K^{+}$channel activity provide evidence for inherent metabolic oscillations in pancreatic $\beta$-cells, Biochem. Biophys. Res. Commun., 205 (1994), pp. 880-885.

[15] M. Düfer, D. Haspel, P. Krippeit-Drews, L. Aguilar-Bryan, J. Bryan, AND G. DREWs, Oscillations of membrane potential and cytosolic $\mathrm{Ca}^{2+}$ concentration in SUR1-/- beta cells, Diabetologia, 47 (2004), pp. 488-498.

[16] G. A. Dunaway, T. P. Kasten, T. Sebo, and R. Trapp, Analysis of the phosphofructokinase subunits and isoenzymes in human tissues, Biochem. J., 251 (1988), pp. 677-683.

[17] L. G. FOE AND R. G. KEMP, Isolation and characterization of phosphofructokinase C from rabbit brain, J. Biol. Chem., 260 (1985), pp. 726-730. 
[18] L. E. Fridlyand, N. Tamarina, and L. H. Philipson, Modeling of $\mathrm{Ca}^{2+}$ flux in pancreatic $\beta$-cells: Role of the plasma membrane and intracellular stores, Am. J. Physiol., 285 (2003), pp. E138-E154.

[19] R. S. HELLER, The comparative anatomy of islets, in Islets of Langerhans, M. S. Islam, ed., Springer, Dordrecht, 2015, pp. 1-18.

[20] J. C. Henquin, H. P. Meissner, And W. Schmeer, Cyclic variations of glucoseinduced electrical activity in pancreatic $\beta$ cells, Pflügers Arch., 393 (1982), pp. 322-327.

[21] J. Keizer And G. Magnus, ATP-sensitive potassium channel and bursting in the pancreatic $\beta$ cell, Biophys. J., 56 (1989), pp. 229-242.

[22] L. L. Kjems, B. M. Kirby, E. M. Welsh, J. D. Veldhuis, M. Straume, S. S. McIntyre, D. Yang, P. Lefèbvre, and P. C. Butler, Decrease in $\beta$-cell mass leads to impaired pulsatile insulin secretion, reduced postprandial hepatic insulin clearance, and relative hyperglucagonemia in the minipig, Diabetes, 50 (2001), pp. 2001-2012.

[23] D. A. Lang, D. R. Matthews, M. Burnett, and R. C. Turner, Brief, irregular oscillations of basal plasma insulin and glucose concentrations in diabetic man, Diabetes, 30 (1981), pp. 435-439.

[24] D. A. Lang, D. R. Matthews, J. Peto, and R. C. Turner, Cyclic oscillations of basal plasma glucose and insulin concentrations in human beings, N. Engl. J. Med., 301 (1979), pp. 1023-1027.

[25] A. V. Matveyenko, D. Liumantara, T. Gurlo, D. Kirakossian, C. D. Man, C. Cobelli, M. F. White, K. D. Copps, E. V. a nd S. Fujita, and P. C. 
Butler, Pulsatile portal insulin delivery enhances hepatic insulin action and signaling, Diabetes, 61 (2012), pp. 2269-2279.

[26] A. V. Matveyenko, J. D. Veldhuis, And P. C. Butler, Measurement of pulsatile insulin secretion in the rat: Direct sampling from the hepatic portal vein, Am. J. Physiol., 295 (2008), pp. E569-E574.

[27] J. P. McKenna, J. Ha, M. J. Merrins, L. S. Satin, A. Sherman, And R. Bertram, $C a^{2+}$ effects on ATP production and consumption have regulatory roles on oscillatory islet activity, Biophys. J., 110 (2016), pp. 733-742.

[28] _ Fast-slow analysis of the integrated oscillator model for pancreatic $\beta$-cells, J. theoret. Biol. (companion paper), (2018).

[29] M. J. Merrins, A. R. V. Dyke, A. K. Mapp, M. A. Rizzo, And L. S. Satin, Direct measurements of oscillatory glycolysis in pancreatic islet $\beta$-cells using novel FRET biosensors for pyruvate kinase M2 activity, J. Biol. Chem., 288 (2013), pp. 33312-33322.

[30] M. J. Merrins, B. Fendler, M. Zhang, A. Sherman, R. Bertram, and L. S. SATIN, Metabolic oscillations in pancreatic islets depend on the intracellular $\mathrm{Ca}^{2+}$ level but not $\mathrm{Ca}^{2+}$ oscillations, Biophys. J., 99 (2010), pp. 76-84.

[31] M. J. Merrins, C. Poudel, J. P. McKenna, J. Ha, A. Sherman, R. Bertram, AND L. S. SATin, Phase analysis of metabolic oscillations and membrane potential in pancreatic $\beta$-cells, Biophys. J., 110 (2016), pp. 691-699.

[32] C. S. Nunemaker And L. S. Satin, Episodic hormone secretion: A comparison of the basis of pulsatile secretion of insulin and GnRH, Endocrine, 47 (2014), pp. 49-63. 
[33] S. O'Rahilly, R. C. Turner, and D. R. Matthews, Impaired pulsatile secretion of insulin in relatives of patients with non-insulin-dependent diabetes, N. Eng. J. Med., 318 (1988), pp. 1225-1230.

[34] A.-M. T. Richard, D.-L. Webb, J. M. Goodman, V. Schultz, J. N. Flanagan, L. Getty-Kaushik, J. T. Deeney, G. C. Yaney, G. A. Dunaway, P.-O. Berggren, And K. Tornheim, Tissue-dependent loss of phosphofructokinase- $M$ in mice with interrupted activity of the distal promoter: impairment in insulin secretion, Am. J. Physiol., 293 (2007), pp. E794-E801.

[35] L. S. Satin, P. C. Butler, J. Ha, and A. S. Sherman, Pulsatile insulin secretion, impaired glucose tolerance and type 2 diabetes, Mol. Aspects Med., 42 (2015), pp. 61-77.

[36] V. Seghers, M. Nakazaki, F. Demayo, L. Anguilar-Bryan, and J. Bryan, Sur1 knockout mice: A model for $K_{A T P}$ channel-independent regulation of insulin secretion, J. Biol. Chem., 275 (2000), pp. 9270-9277.

[37] P. Smolen, A model for glycolytic oscillations based on skeletal muscle phosphofructokinase kinetics, J. theor. Biol., 174 (1995), pp. 137-148.

[38] P. Smolen and J. KeIzer, Slow voltage inactivation of $\mathrm{Ca}^{2+}$ currents and bursting mechanisms for the mouse pancreatic $\beta$-cell, J. Membrane Biol., 127 (1992), pp. 9-19.

[39] S. H. Song, S. S. McIntyre, H. Shah, J. D. Veldhuis, P. C. Hayes, and P. C. Butler, Direct measurement of pulsatile insulin secretion from the portal vein in human subjects, J. Clin. Endocrinol. Metab., 12 (2000), pp. 4491-4499. 
[40] J. Sturis, E. Van Cauter, J. D. Blackman, and K. S. Polonsky, Entrainment of pulsatile insulin secretion by oscillatory glucose infusion, J. Clin. Invest., 87 (1991), pp. $439-445$.

[41] K. ToRnheim, Are metabolic oscillations responsible for normal oscillatory secretion?, Diabetes, 46 (1997), pp. 1375-1380.

[42] K. Tornheim and J. M. Lowenstein, The purine nucleotide cycle. III. Oscillations in metabolite concentrations during the operation of the cycle in muscle extracts, J. Biol. Chem., 248 (1973), pp. 2670-2677.

[43] M. Watts, B. Fendler, M. J. Merrins, L. S. Satin, R. Bertram, and A. Sherman, Calcium and metabolic oscillations in pancreatic islets: Who's driving the bus?, SIAM J. Appl. Dyn. Syst., 13 (2014), pp. 683-703.

[44] G. C. Yaney, V. Schultz, B. A. Cunningham, G. A. Dunaway, B. E. Corkey, And K. Tornheim, Phosphofructokinase isozymes in pancreatic islets and clonal $\beta$ cells (INS-1), Diabetes, 44 (1995), pp. 1285-1289.

[45] M. Zhang, P. Goforth, R. Bertram, A. Sherman, and L. Satin, The $C a^{2+}$ dynamics of isolated mouse $\beta$-cells and islets: Implications for mathematical models, Biophys. J., 84 (2003), pp. 2852-2870. 